Dominique Andolfatto, Université de Bourgogne Franche-Comté

Dominique.Andolfatto@u-bourgogne.fr

Dominique Labbé, Pacte-CNRS, Institut d'études politiques de Grenoble

dominique.labbe@umrpacte.fr

\title{
La réforme, la rue et les syndicalistes
}

\section{Résumé}

En 2016, une nouvelle réforme du droit du travail a illustré les faiblesses des relations sociales en France. Le gouvernement a agi sous contrainte, sans annoncer ses objectifs et a semblé hésiter. La capacité de mobilisation des syndicats a été faible. Leurs effectifs, leurs implantations et leur audience électorale continuent à diminuer. Les nouvelles conventions d'entreprise apparaissent déséquilibrées en défaveur des travailleurs.

\begin{abstract}
In 2016, a new labour law reform illustrated the weaknesses of French industrial relations. The government acted hesitantly and under constraint, without announcing its objectives. Trade unions were unable to organize significant protests. Their membership, their presence in the work-place and their electoral audience continue to decline. The new contracts appear to be unbalanced and not in the interests of the workers.
\end{abstract}

Article paru dans la revue Commentaire. 156, Hiver 2016-2017, p. 425-432.

Manuscrit des auteurs. Toute citation doit se faire à partir de la revue. 
Au printemps 2016, la France a connu une agitation confuse qui semble devenir coutumière. Après les réformes sur la sécurisation de l'emploi (2013), la formation professionnelle (2014) et le dialogue social (2015), sans effets réels sur l'emploi et les relations sociales à la française, le gouvernement a cherché à lancer une réforme a priori plus volontaire et ambitieuse. Etonnamment, il n'a pas voulu annoncer clairement son ambition et ses objectifs et il a donné le sentiment d'agir sous contrainte, en hésitant et en tâtonnant. La contestation que cette loi a provoquée donne l'occasion de faire le point sur l'état des forces syndicales en France. Plus au fond, ce sont aussi les relations industrielles à la française qu'il s'agit d'interroger.

\section{Une réforme sous contrainte}

La réforme, intitulée pompeusement "Nouvelles libertés et nouvelles protections pour les entreprises et les actifs" puis, plus sobrement, "Travail" est intervenue sous pression.

D'abord, la montée irrépressible du chômage : celui-ci culmine à 2,9 millions de sansemploi (au sens du BIT) au premier semestre 2015, soit un taux de chômage de 10,1 \% de la population active en métropole (et 300000 chômeurs en plus depuis les élections de 2012).

De plus, en 2014, la Commission européenne a placé la France sous surveillance étroite, en raison de l'aggravation de sa dette publique, d'un manque de compétitivité économique, d'un coût du travail jugé trop élevé... En conséquence, lors de préconisations récurrentes, il est exigé de la France une révision du cadre juridique régissant le contrat de travail, de donner "aux employeurs [des] possibilités pour déroger aux accords de branche", de leur permettre de "moduler leurs effectifs en fonction de leurs besoins". Ainsi, les entreprises doivent pouvoir "déterminer, de façon flexible, au cas par cas et après négociations avec les partenaires sociaux, s'il y a lieu de déroger à la durée légale du travail de 35 heures par semaine". De même, il est demandé de revoir la loi relative à la sécurisation de l'emploi de 2013, qui a produit trop peu de résultats et "de donner plus de latitude aux entreprises pour adapter les salaires et le temps de travail à leur situation économique"1.

Enfin, le gouvernement est aussi soumis aux pressions contradictoires des organisations patronales et syndicales, pour ne pas parler des électeurs qui ont lourdement sanctionné l'exécutif lors de toutes les élections intermédiaires depuis 2012, ou du président de la

\footnotetext{
${ }^{1}$ Recommandation du Conseil de l’Union Européenne, 13 mai 2015 puis 18 mai 2016.
} 
République qui a lié son sort non pas à une amélioration substantielle de l'emploi mais à un indice statistique : "l'inversion de la courbe du chômage".

A la rentrée de 2015, un bouquet de rapports rendus publics reprend ou préconise de nombreuses pistes concernant la réforme du code du travail, la négociation collective, la digitalisation des relations sociales ${ }^{2} \ldots$ Cela dit, ces documents prennent rarement appui sur des audits précis, procèdent par induction, s'avèrent assez abstraits ou ne concernent que les plus grandes entreprises de sorte qu'aucune réforme ne se dessine clairement. La conférence sociale, du 19 octobre 2015, qui réunit les pouvoirs publics et les partenaires sociaux, a pour ordre du jour des sujets assez techniques (le projet du compte personnel d'activité) ou spéculatifs : la "transformation numérique", la "croissance verte". Une réforme du droit et des relations du travail serait-elle trop clivante, complexe ou dérangeante, compte tenu des représentations ou des rentes de situation qui prédominent?

Mais c'est déjà suffisant pour que la commission exécutive de la CGT - suivant son nouveau secrétaire général, Philippe Martinez dont le mandat doit être encore validé par le congrès confédéral d'avril 2016 - décide de boycotter la conférence, n’y voyant qu'une opération de "communication" et une "grande messe d'experts patronaux"3.

De son côté, la direction du Medef escompte bien obtenir des réformes qu'elle estime majeures : remise en cause d'une "fiscalité trop lourde" pour les entreprises ; critique de la loi Rebsamen sur le dialogue social (2015) qui "n'est (...) pas à la hauteur des transformations nécessaires (...) alors que nous devons faire en sorte que le dialogue social au plus près du terrain, c'est-à-dire dans l'entreprise, devienne la source principale des règles sociales qui s'y appliquent" ; nécessité d'un "plafonnement des indemnités prud'homales [pour] sécuriser la rupture du contrat de travail par le salarié comme pour l'employeur [et donc] faciliter l'embauche"4.

François Hollande, lui-même, paraît décidé à sortir du cadre étroit de l'ordre du jour de la conférence sociale et, d'emblée, il déclare vouloir "réinventer notre modèle social". Il

\footnotetext{
${ }^{2}$ J.-D. Combrexelle, La négociation collective, le travail et l'emploi, rapport au Premier ministre, Paris, France-Stratégie, sept. 2015 ; B. Mettling, Transformation numérique et vie au travail, rapport à l'attention de M. El Khomri, ministre du Travail, de l'Emploi, de la Formation professionnelle et du Dialogue social, sept. 2015 ; R. Badinter et al., Définir les principes essentiels du droit du travail, rapport au Premier ministre, janv. 2016.

${ }^{3}$ Interview de P. Martinez, Le journal du dimanche, 18 oct. 2015.

${ }^{4}$ Discours de P. Gattaz lors de la conférence sociale du 19 oct. 2015. Précisions que le Conseil constitutionnel a censuré le 6 août 2015 une première tentative de plafonnement des indemnités prud'homales en cas de licenciement sans cause réelle et sérieuse prévue dans la loi pour la croissance, l'activité et l'égalité des chances économiques (dite Macron) du 6 août 2016.
} 
affirme : "le statu quo n'est plus possible et l'alternative c'est la réforme ou la rupture". Et il annonce : "L'objectif, ce n'est pas d'inverser la hiérarchie des normes, c'est faire le pari de la confiance pour que ce soient les employeurs et les salariés, à travers leurs représentants, qui déterminent d'un commun accord les solutions les meilleures pour les entreprises et pour leur branche professionnelle". Manifestement, F. Hollande a déjà la loi Travail en tête et semble déterminé à renoncer aux textes cosmétiques. Pourtant, quelques mois plus tard, les partenaires sociaux, comme le grand public, découvrent la réforme grâce à des fuites dans la presse ! Etonnante fabrique de la réforme que justifie en réalité l'échec de la négociation sur le dialogue social, début 2015 et le veto a priori - pour des raisons de principe ou de

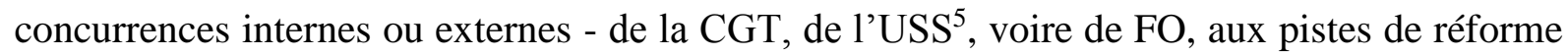
esquissées à l'automne $2015^{6}$.

Une partie de la contestation du printemps 2016 s'explique par l'élaboration de la loi Travail, dans le secret des cabinets, qui a pu donner le sentiment que le gouvernement cherchait à cacher ses véritables objectifs. Mais la faible portée de cette contestation apporte surtout une nouvelle illustration de la faiblesse et des divisions syndicales.

\section{Faiblesse de la mobilisation}

Le projet de loi Travail suscite immédiatement une levée de boucliers : "recul historique des droits des salariés" selon la CGT, "révolution libérale" selon FO, réforme "sur-mesure pour le patronat" selon l'USS ${ }^{7}$. D'autres positionnements sont plus nuancés : "des avancées et des inquiétudes" selon la CFDT, un projet "très déséquilibré entre flexibilité et sécurité" selon la CFE-CGC (et la CFDT), un projet "qui doit évoluer pour plus de sécurisation pour les salariés" selon la $\mathrm{CFTC}^{8}$. Une coalition se forme entre les diverses organisations pour faire

\footnotetext{
${ }^{5}$ Union Syndicale Solidaires, associant notamment les syndicats SUD.

${ }^{6}$ Curieusement, le Conseil d'Etat a considéré que le gouvernement avait bien informé les partenaires sociaux de ses intentions, conformément à l'article 1 du code du travail qui impose de proposer à ces derniers de négocier entre eux, et avant son passage au parlement, tout projet de réforme concernant les relations du travail et l'emploi. Cela serait intervenu après le rapport Combrexelle eut été rendu piblic. Cependant, les partenaires n'auraient pas souhaité s'emparer du projet (avis du Conseil d'Etat, 17 mars 2016).

${ }^{7}$ Communiqué de la CGT, 19 fév. 2016 ; éditorial de J.-C. Mailly, FO Hebdo, 24 fév. 2016, tract de l'USS, 29 fév. 2016.

${ }^{8}$ Communiqué de la CFDT, 18 fév. 2016 ; interview de L. Berger dans Le Monde, 20 février 2012 ; communiqué de C. Couvert, présidente de la CFE-CGC, 23 fév. 2016 ; communique de la CFTC, 22 février 2016.
} 
échec à certaines dispositions et, d'abord, au plafonnement des indemnités prud'homales. Si la concertation qui s'ouvre apporte finalement satisfaction à la CFDT, à la CFTC, voire à la CGC - surtout absorbée par la préparation de son congrès et le remplacement de sa présidente -, les autres organisations exigent le retrait pur et simple de la réforme et vont multiplier les mobilisations. Quelque 13 manifestations se succèdent du 9 mars au 5 juillet 2016 avant de reprendre le 15 septembre $2016 \ldots$ La contestation semble ainsi traduire une fracture du mouvement syndical, au niveau national, opposant un camp d'opposants irréductibles (rejoint par FO) à un camp plus réformiste. En réalité, du fait de la professionnalisation du syndicalisme, les pratiques des uns et des autres demeurent assez proches.

Le mode d'action collective retenu tend à réduire la manifestation un simple rituel, parfois spectaculaire mais sans efficacité, sauf à affirmer certaines identités minoritaires, à commémorer un passé révolu, à l'instar d'autres recours à des manifestations ces dernières années, notamment dans les pays du sud de l'Europe, pour dénoncer des politiques dites d'austérité.

On dispose en effet de données assez précises pour évaluer le nombre de manifestants. Certes les écarts peuvent apparaître importants selon les sources : les chiffres présentés par les syndicats sont 2 à 10 fois supérieurs à ceux produits par le ministère de l'Intérieur (voire le graphe ci-dessous).

Cela dit en 2015, une expertise réalisée par la sociologue Dominique Schnapper, l'inspecteur général de l'INSEE Pierre Muller et le politologue Daniel Gaxie, de l'Université de Paris I, en relation avec la police mais aussi les organisations syndicales, avait conclu au bien-fondé de la méthode de comptage et des chiffres de la police, ceux-ci étant même parfois légèrement surestimés ${ }^{9}$. Si la même analyse vaut pour tous les services de police du territoire, les chiffres produits par le ministère de l'Intérieur lors des journées nationales de manifestation sont donc crédibles. Or, en 2016, seule une journée de manifestations a rassemblé 400000 personnes (le 31 mars), deux (dont cette dernière) ont atteint ou dépassé les 200000 personnes, la moyenne s'établissant à 125000 personnes (dont 20000 à Paris). Si ces chiffres sont loin d'être négligeables, ils restent tout de même modestes au regard du nombre de salariés et, plus étonnant encore, du nombre de syndiqués revendiqués par les organisateurs des manifestations. Au plus $10 \%$ d'entre eux auraient suivi les consigne de leur

\footnotetext{
${ }^{9}$ Rapport de la commission de réflexion sur la mesure du nombre des participants aux manifestations de rue, 31 mars 2015.
} 
syndicat et, sans doute moins, compte tenu de la participation d'étudiants, voire de lycéens à ces manifestations, à tout le moins aux premières d'entre elles, lorsque l'année universitaire n'était pas encore terminée.

\section{La participation aux manifestations contre la loi Travail au printemps 2016}

(sources : ministère de l'Intérieur et syndicats ; échelle en milliers de manifestants).

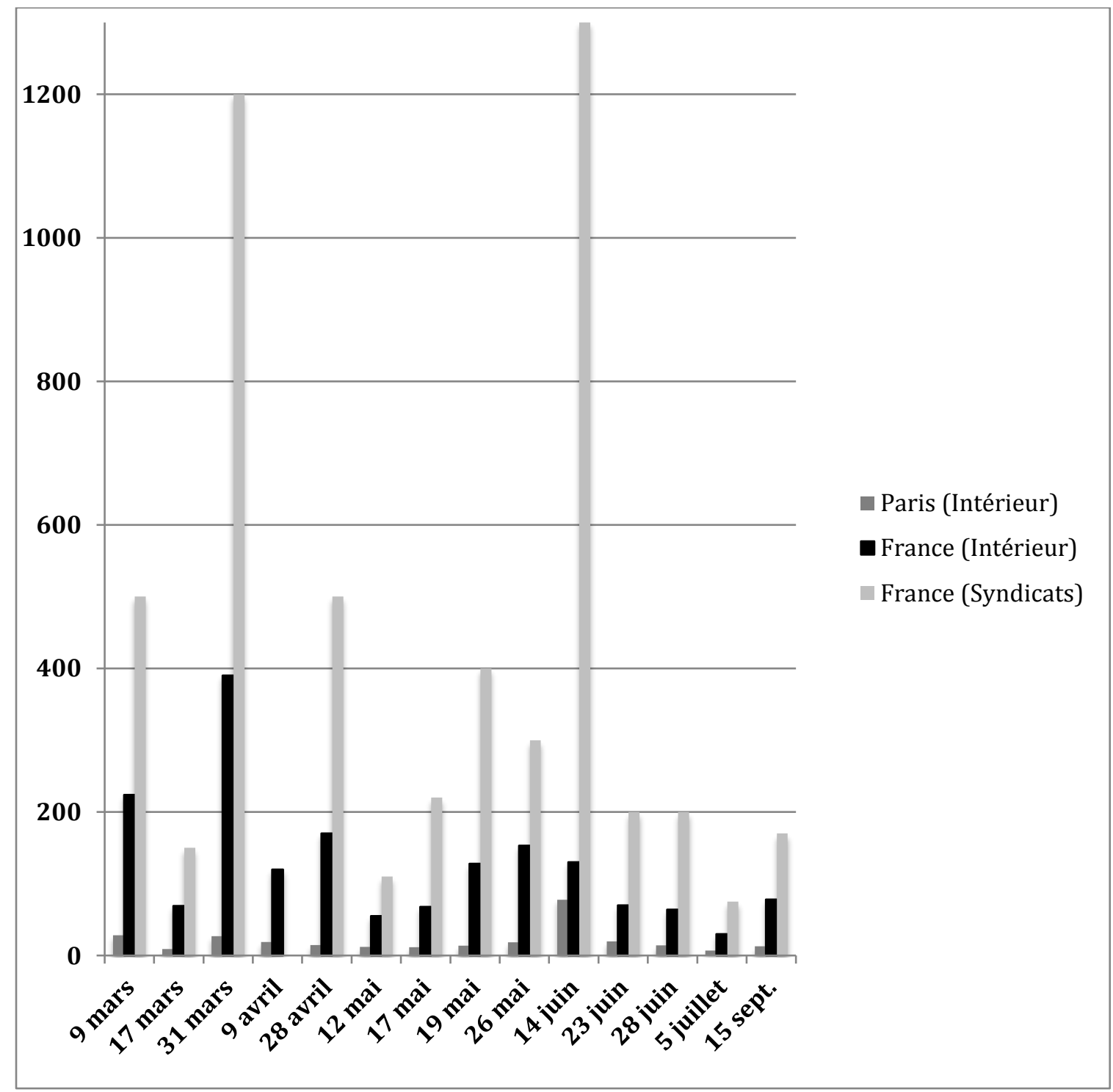

Plus au fond, cette faiblesse relative et l'échec de ces actions interrogent sur les capacités de mobilisation des syndicats et leur situation réelle.

Faiblesse des forces syndicales 
Toutes les fédérations et confédérations connaissent leurs syndiqués puisqu'elles sont capables de leur envoyer au moins une fois par an un certificat leur permettant de déduire la cotisation de leurs impôts. Ces effectifs, jalousement tenus secrets, sont connus indirectement grâce à une série d'indices : montant des cotisations collectées, mandats aux congrès, tirage des bulletins syndicaux, élections professionnelles, etc. Il y a une dizaine d'années, nous avons reconstitué ce puzzle ${ }^{10}$. En tout, il y avait en France 1,7 millions de syndiqués. Seule la CGT atteignait le demi million et la CFDT dépassait les 400000.

Quant au nombe de contribuables qui demandent la déduction, il y a une dizaine d'années, ils étaient moins de 1,5 millions, corroborant ainsi les résultats de notre enquête. On peut d'ailleurs se demander pourquoi le ministère des Finances a renoncé à publier ces données. On sait tout de même qu'en 2011, ces déductions ont représenté 130 millions d'euros ${ }^{11}$.

Certes, au printemps 2016, le ministère du Travail a publié une bonne nouvelle : 11,2\% de syndiqués ${ }^{12}$. Cette enquête par sondage étant unique, il est impossible de redresser le taux d'embellissement inévitable dans ce genre d'opération. D'ailleurs, rien ne vient corroborer ce miracle. En effet, depuis 10 ans, aucune organisation n'a revendiqué une augmentation du nombre de ses adhérents. Récemment les confédérations CGT et CFDT ont même reconnu un tassement du montant des cotisations encaissées depuis 2010-2011 et une baisse de quelques milliers du nombre de leurs adhérents.

D'après l'INSEE, il y a 23,9 millions de salariés en France, le taux de syndicalisation est donc de 7,1 pour 100 salariés, taux pratiquement inchangé depuis notre enquête de 2007. C'est le taux le plus bas de tous les grands pays industriels.

Depuis 2008 la thèse officielle sinon dominante est que, certes, les syndicats n'ont plus beaucoup d'adhérents mais que cela n'a guère d'importance puisqu'ils ont des électeurs et que ces électeurs suffisent pour fonder leur représentativité. Il a été objecté que les entreprises de moins de 11 salariés n'ont aucune élection. En décembre 2012, il a été organisé un scrutin pour ces salariés. Sur les 4,7 millions d'inscrits, moins de 490000 ont exprimé un vote $(10,4 \%)$. Cet échec n'est pas un accident ou le produit d'une organisation inadaptée, comme

${ }^{10}$ D. Andolfatto, D. Labbé, Les syndiqués en France. Qui ? Combien ? Où ?, Rueil-Malmaison, Editions Liaisons, 2007. Notre enquête concluait à un taux de syndicalisation de 7,2\% des salariés.

${ }^{11}$ M. Chevreux, C. Darmaillacq, "La syndicalisation en France : paradoxes, enjeux et perspectives", Trésor-Eco, n 129, mai 2014, p. 8.

${ }^{12}$ M. Pignoni, "La syndicalisation en France. Des salariés deux fois plus syndiqués dans la fonction publique", Dares analyses, $n^{\circ} 025$, mai 2016. Le taux est calculé par rapport aux seuls salariés qui ont un emploi, ignorant les chômeurs habituellement intégrés dans le calcul des taux de syndicalisation, ce qui conduit à gonfler le taux et interdit toute comparaison (ce dont se garde la note). 
on l'a dit à l'époque. Partout, les taux de participation aux élections professionnelles déclinent, y compris dans les trois fonctions publiques et les grandes entreprises nationales.

Environ 18,5 millions de salariés sont concernés par la loi Travail (les entreprises soumises au droit des conventions collectives quel que soit leur statut). Parmi ces salariés, moins de 13 millions se voient offrir la possibilité de voter et seulement 5 millions apportent un suffrage à une organisation syndicale, soit moins de $40 \%$ des électeurs et de $27 \%$ des salariés concernés.

La faiblesse du syndicalisme français se marque d'abord par cette difficulté à organiser des scrutins et à amener les salariés aux urnes. Cette caractéristique est d'autant plus frappante que les moyens mis à leur disposition sont considérables. Par exemple, le scrutin de décembre 2012 dans les TPE a officiellement coûté 20 millions d'euros (impression de la propagande et du matériel électoral, envoi de ce matériel aux électeurs, dépouillement), soit plus de 20 euros le vote!

En tous cas, il semble difficile de prétendre qu'avec de tels taux de syndicalisation et de participation, la représentativité des syndicats ne pose aucun problème !

On a alors répondu que le bon critère est celui de la présence syndicale dans les entreprises et les administrations. Après tout, peu importe que les adhérents et les électeurs se dérobent puisque des institutions existent et qu'elles sont capables de fonctionner au service des salariés.

La meilleure mesure de l'implantation syndicale dans une entreprise ou une administration, c'est sa capacité à présenter une liste aux élections professionnelles (CE et DP pour les entreprises privées, commissions paritaires pour la fonction publique). Pour les entreprises concernées par la loi Travail, il n'y a aucune difficulté technique à calculer ces taux puisque les résultats des élections sont centralisés au titre de la mesure de la représentativité. Ces calculs avaient été fait dans les années 1990 et poursuivis irrégulièrement jusqu'en $2004^{13}$. A l'époque, il apparaissait que, pour les établissements de plus 50 salariés, un salarié sur deux disposait d'au moins une équipe syndicale dans son établissement et que les élections concurrentielles (au moins deux listes) concernaient moins du tiers des salariés (pour la plupart employés dans de grands établissements). Depuis 2004, ces calculs sont abandonnés au profit de l'enquête "Reponse" - piloté par le ministère du Travail - qui donnait des résultats plus favorables (voir le tableau ci-dessous).

\footnotetext{
${ }^{13} \mathrm{R}$. Matuszewicz et D. Boulmier, "L'audience électorale du syndicalisme à l'heure de la réforme de la représentativité", D. Andolfatto (dir). Les syndicats en France, Paris, La Documentation française, 2013, p 132-133.
} 
L'évolution de la présence syndicale dans les entreprises

\begin{tabular}{|l|c|c|c|c|c|}
\hline \multirow{2}{*}{} & \multicolumn{4}{|c|}{$\begin{array}{c}\text { \% des établissements de 50 salariés et plus } \\
\text { (et, entre parenthèses, \% des établissements }\end{array}$} & $\begin{array}{c}\text { \% des salariés concernés } \\
\text { de 20 salariés et plus) } \\
\text { de établissements }\end{array}$ \\
\hline & $1992-93$ & $1998-99$ & $2004-05$ & $2010-11$ & $2009-2012$ \\
\hline CGT & 28 & $32(16)$ & $35(19)$ & $(17)$ & 67 \\
\hline CFDT & 26 & $31(17)$ & $36(19)$ & $(18)$ & 69 \\
\hline FO & 20 & $22(11)$ & $25(13)$ & $(11)$ & 55 \\
\hline CFTC & 9 & $9(5)$ & $14(7)$ & $(5)$ & 40 \\
\hline CGC & 14 & $14(7)$ & $16(8)$ & $(6)$ & 26 \\
\hline UNSA & - & - & - & - & 16 \\
\hline USS & - & - & - & - & $10(* * *)$ \\
\hline Autres $(* *)$ & - & $4(4)$ & $8(3)$ & $(4)$ & \\
\hline Total & 54 & 58 & 63 & 59 & 20 \\
\hline
\end{tabular}

(**) UNSA, USS et autres.

(***) Hors UNSA et USS.

Sources : ministère du Travail (enquêtes "Reponse") et Les Echos, 13 nov. 2012.

Il n’y a aucun moyen de contrôler les déclarations des enquêtés et ce sont les employeurs qui donnent aux enquêteurs les noms et les coordonnées des syndicalistes présents dans leur entreprise. Les syndicats eux-mêmes n'invoquent guère ces résultats comme si la mariée était trop belle. Par exemple, lors de son congrès de 2016, la CGT a précisé ne rayonner que sur un quart du salariat. Ce manque de crédibilité a-t-il conduit le ministère à ne pas publier les résultats de la dernière enquête qui, au surplus, faisait état d'un net tassement de toutes les confédérations sur les dix dernières années?

Tous les indices convergent donc vers le constat - déjà dressé depuis plusieurs années d'un affaiblissement continu des syndicats ${ }^{14}$. Or malgré cette situation, tous les gouvernements, quelle que soit leur couleur politique, prétendent développer le dialogue social. La loi Travail élargit de nouveau le champ de la négociation d'entreprise. Tout le

\footnotetext{
${ }^{14}$ Voir notamment D. Andolfatto, D. Labbé, "Syndicats : des mythes aux réalités", Commentaire, $\mathrm{n}^{\circ} 130,2010, \mathrm{p} .379-386$.
} 
monde fait semblant d'ignorer que, pour négocier il faut être au moins deux et que, en l'occurrence, l'un des deux acteurs est absent ou se trouve dans un grand état de faiblesse. Cela conduit logiquement à se demander si, lors de ces négociations, les syndicalistes ont réellement le pouvoir de dire non.

\section{Le pouvoir de dire non?}

Depuis de nombreuses années, les réformes successives du code du travail - dont la dernière loi de 2016 - tendent donc à faire de l'entreprise le lieu privilégié où s'élaborent les règles du travail. Sont notamment invoqués la nécessité d'adaptations rapides des entreprises, le maintien et le renforcement de leur compétitivité dans le contexte de globalisation. Toutes les confédérations ont accompagné voire souhaité ce processus. Même la CGT, pourtant à l'avant-garde de l'opposition à la loi El Khomri, l'a légitimé en signant, avec la CFDT, le Medef et la CGPME, la "position commune" de 2008, à l'origine de la refondation de la représentativité syndicale sur les résultats des élections professionnelles au niveau des entreprises. Seule la confédération FO affiche une réticence continue, de crainte d'une "inversion des normes" et d'affaiblissement du pouvoir syndical, mais bon nombre de ses équipes sont de longue date très impliquées dans la négociation collective, à quelque niveau que ce soit.

On sait peu de choses de cette négociation d'entreprise mais quelques indices permettent de douter de sa nature sinon de sa réalité. Ainsi le taux de signature des accords négociés dans les entreprises est extrêmement élevé (nombre de fois qu'un syndicat signe un accord lorsqu'il est présent dans l'entreprise). En 2014 - dernières données disponibles ${ }^{15}$-, les organisations syndicales ont signé entre $84 \%$ (CGT) et $92 \%$ (CFDT) des accords d'entreprise qu'elles ont négociés. Autant dire que les clivages entre organisations cèdent pour l'essentiel à ce niveau, ce qui n'est pas le cas au niveau des branches, où se remarquent les refus de signature de la CGT, voire secondairement de FO. A moins de considérer qu'au niveau des entreprises, les syndicats obtiennent quasiment toujours satisfaction, il faut admettre que - à part dans quelques grands établissements - les syndicalistes ne sont pas en capacité de dire "non" aux demandes de leurs employeurs. Quoi de plus logique ? Le délégué syndical (DS) est salarié de l'entreprise. Pour son emploi et sa carrière, il dépend du

\footnotetext{
${ }^{15}$ En sept. 2016, le ministère du Travail n'a pas publié le bilan de la négociation collective pour 2015, contrairement à un usage constant depuis 1983. Nos questions pour connaître la date de publication de ce bilan sont restées sans réponse.
} 
responsable des ressources humaines en face duquel il s'assoit pour négocier. De plus, l'essentiel des moyens de la section syndicale est fourni par l'employeur. Etant donné le petit nombre des adhérents, la solitude du DS, sa dépendance matérielle et intellectuelle vis-à-vis de sa hiérarchie rendent difficile un refus de signature. La situation serait sans doute assez différente si le négociateur syndical était un salarié du syndicat, rompu à la négociation, appuyé par un grand nombre d'adhérents et indépendant de l'employeur, comme c'est le cas dans la plupart des grands pays industriels.

On connait mal ce qui est signé, d'autant plus que ces textes font rarement l'objet de publicité (ce que la loi de 2016 devrait en principe changer via un décret encore à venir). Quelques accords ont toutefois été rendus publics au cours des derniers mois et permettent d'éclairer les relations entre les syndicats et les employeurs au niveau des entreprises et, plus au fond, d'illustrer cette "inversion des normes" qui a beaucoup polarisé les débats sur le projet de loi Travail.

Par exemple, chez Peugeot, en juillet 2016, était signé - sauf par la CGT - un nouvel accord d'entreprise : "Construire ensemble l'avenir du Groupe : un Nouvel Elan pour la Croissance". Il s'agit d'un très long texte (plus de 100 pages), souvent verbeux, parfois obscur (de ce point de vue, on est bien loin d'une simplification du code du travail parfois annoncée dans les débats publics). Le texte n'est pas organisé en articles, paragraphes et alinéas numérotés, mais écrit comme une gigantesque dissertation dans laquelle sont noyées les quelques dispositions cruciales mais difficiles à retrouver et à interpréter.

Prenons deux sujets : le temps de travail et l'emploi. La loi de 2016, après celle de $2008^{16}$, autorise de nombreuses dérogations concernant le temps de travail. En se fondant sur cette dernière, la direction de Peugeot a obtenu une grande flexibilité : le temps de travail est annualisé et pourra fluctuer entre deux bornes par rapport à l'horaire contractuel annuel, soit pour un temps plein entre : -12 jours (ou -84 heures) et +8 jours (ou +56 heures). Enfin, les horaires peuvent être constamment modifiés avec un préavis de 48 heures. Les modulations sont appréciées à la fin de chaque mois et donnent lieu au paiement d'heures supplémentaires en cas de dépassement de la borne supérieure.

L'accord fixe également des objectifs d'embauche : "2 000 emplois jeunes" par an (6 000 en 3 ans) et un "objectif" de 1000 emplois en CDI sur la période de validité de la convention (3 ans et donc 333 CDI par an). Aucun détail n'est donné sur le nombre de départs annuels

\footnotetext{
${ }^{16}$ Loi Travail et loi "portant rénovation de la démocratie sociale et réforme du temps de travail" du 20 août 2008.
} 
"naturels" attendus. Etant donné les effectifs du groupe en France (79 000 salariés en France sur 184 000), il y aura au minimum 2000 départs par an. Ces départs sont apparemment compensés par des emplois jeunes. Mais ces emplois sont à durée déterminée. Les embauches en CDI ("objectif" 333 par an) ne compenseraient donc, au maximum, que $17 \%$ des départs (1 000 emplois sur 6000). L'accord prévoit que les "jeunes" représenteront $50 \%$ des nouveaux CDI, ce qui signifie que moins d'un "emploi jeune" sur 10 (500 sur 6 000) a une chance de se voir recruter en CDI. Au total, c'est donc une déflation très forte des effectifs "stables" qui est consentie par les syndicats (rien n'est dit sur l'intérim et les CDD).

Etait-il possible pour les syndicalistes, autres que la CGT, de refuser de signer ? Pourquoi personne n'a soulevé publiquement la question des effectifs ? Bien sûr, on peut considérer que les préretraites, la flexibilité des horaires et la contraction de l'emploi sont le prix à payer pour maintenir les sites français de l'entreprise. Mais dès lors, ce qu'on appelle la démocratie sociale ne semble qu'un artifice, un théâtre, qui n'a guère de prise sur une réalité que l'on préfère laisser dans le flou. Cela est d'autant plus remarquable que l'accord affirme vouloir "améliorer l'attractivité du syndicalisme" !

L'accord Solvay France (près de 6000 salariés dans la chimie) éclaire un autre aspect du débat sur la négociation d'entreprise. Signé le 31 mai 2016 par la CGC et la CFDT (mais non la CGT), il est relatif à la "rénovation du dialogue social". Il est intervenu consécutivement à la loi Rebsamen du 17 août 2015 qui permet un regroupement conditionnel des instances représentatives du personnel et, en l'occurrence, il conduit à la disparition des délégués du personnel au niveau local. Une institution unique regroupera leurs attributions avec celles du CHSCT. Elle prend pour nom CHSECTDD : comité d'hygiène, sécurité, environnement, conditions de travail, développement durable. Les délégués syndicaux (DS) demeurent les interlocuteurs de l'employeur pour la négociation collective.

Ce réaménagement fait partie d'un projet - a priori volontariste et ambitieux - visant à promouvoir un "dialogue social de qualité [vu comme] une des conditions de la performance économique de l'entreprise" et à "valoriser l'action des représentants du personnel" ${ }^{17}$. En effet, le taux de syndicalisation dans l'entreprise est très faible : $5 \%$ selon le DRH, avec des équipes syndicales qui peinent à se renouveler. A brève échéance, cela met en cause l'élaboration et la signature des accords d'entreprise dont la portée sera décisive, sans compter toutes les obligations légales concernant le dialogue social. Dès lors, Solvay déploie beaucoup de moyens pour maintenir les organisations syndicales en survie et, si possible, relancer une

\footnotetext{
${ }^{17}$ Communiqué du Groupe Solvay, 2 juin 2016.
} 
dynamique syndicale. En plus des heures de délégations et des décharges horaires pour la préparation et la participation aux instances représentatives du personnel, Solvay créé des "coordinateurs syndicaux", parallèlement aux "délégués syndicaux centraux" (qui bénéficient des mêmes décharges pouvant aller jusqu'au plein temps). Des "moyens matériels" (locaux, bureautique, informatique, téléphonie...) et des "moyens en temps" sont également mis à disposition des équipes au niveau central et au niveau local. Au total, ce sont au bas mot l'équivalent d'une quinzaine de personnes à temps plein qui, au sein de l'entreprise, pourront se consacrer à l'activité syndicale (trois syndicats étant représentatifs au niveau du groupe). Autrement dit, l'entreprise financera un poste de syndicaliste par fraction de 300 à 400 salariés. Ces "permanents" bénéficieront d'un dispositif spécifique garantissant l'évolution de leur rémunération et de leur carrière. Enfin, chaque organisation représentative bénéficiera d'une subvention annuelle de 10574 euros. Ces décharges et subventions s'ajoutent aux institutions légales prises en charge par l'entreprise.

Reste à soutenir la syndicalisation et c'est ici que l'accord Solvay se montre le plus avantgardiste. Un organisme extérieur à l'entreprise prendra directement en charge la part des cotisations des adhérents qui échappe à la déduction fiscale. Pour les salariés, l'adhésion syndicale deviendra donc gratuite. L'accord veut ainsi favoriser "l'écoute et l'information des salariés" et, implicitement, en finir avec un syndicalisme hors sol. Mais cette politique se justifie aussi "compte tenu des perspectives démographiques de départ des militants".

Evidemment on se félicite de voir des employeurs faire preuve de tant de sollicitude à l'égard du syndicalisme. On a tout de même l'impression d'une démocratie sociale à l'envers : on construit d'abord un système institutionnel complexe et coûteux puis on cherche à lui donner artificiellement une base sociale pour en assurer l'existence et la légitimité.

\section{Conclusion}

Concernant les relations sociales et la syndicalisation, on peut s'interroger d'abord sur la "fébrilité statistique" qui s'est emparé du ministère du Travail : publication aléatoire des données collectées, non-publication d'enquêtes, changements de base de calcul qui entraînent des ruptures dans les séries, invention de nouveaux indices sans réelle consistance... Tout cela traduirait-il le désarroi d'une administration à qui l'on demande de bâtir des "villages Potemkine", au lieu de fournir les éléments essentiels de connaissance nécessaires à l'action publique et à l'information des citoyens? 
Quant au fond, depuis longtemps, les pouvoirs publics disent: "les entreprises peuvent s'affranchir de la rigidité du cadre légal en négociant avec leurs syndicats des accords adaptés à leur situation". La loi El Khomri ne fait qu'aller plus avant dans cette direction. Pour la majorité des entreprises, cela ne fait que déplacer le problème. Elles n'ont pas les moyens de Solvay ou de Peugeot et ne peuvent susciter, plus ou moins artificiellement, des interlocuteurs syndicaux quand ils n'existent pas ou qu'ils ne sont pas en capacité de prendre des engagements au nom des salariés qu'ils sont censés représenter ou qu'ils ne souhaitent pas le faire pour des raisons électorales évidentes.

Dès lors, renvoyer la responsabilité de la déréglementation du travail au niveau des entreprises est assez hypocrite. Etant donné la faiblesse du syndicalisme en France, les résultats risquent d'être de nouveau limités et décevants. Or la situation actuelle du syndicalisme français est surtout un effet, non désiré mais logique, des politiques publiques poursuivies en ce domaine depuis un demi-siècle. Tous les gouvernements et les responsables des grandes entreprises ont voulu aider les syndicats qu'ils considéraient comme des acteurs indispensables à la modernisation de l'économie française. Pour cela, ils leur ont accordé un grand nombre d'aides et de privilèges (à la manière de Solvay) qui ont permis aux syndicalistes de se passer d'adhérents. Le résultat final est à l'opposé du but poursuivi : affaiblissement continu, représentativité problématique, blocage de tous ordres et sentiment d'abandon chez de nombreux salariés, conduisant à de brusques flambées de colère et de violence. Ne serait-il pas temps de questionner ces politiques?

Malgré tout, il reste dans de nombreuses entreprises et administrations des syndicalistes qui se dévouent quotidiennement pour aider leurs collègues de travail à résoudre les problèmes quotidiens. Ces militants sont la preuve qu'un renouveau du syndicalisme français est toujours possible si les pouvoirs publics et les employeurs voulaient bien reconsidérer les politiques suivies depuis près d'un demi-siècle. 